\title{
A SIMULATION MODEL TO IMPROVE WAREHOUSE OPERATIONS
}

\author{
Jean Philippe Gagliardi \\ Jacques Renaud \\ Angel Ruiz \\ Faculté des sciences de l'administration \& CIRRELT \\ Université Laval \\ Québec, G1K 7P4, CANADA
}

\begin{abstract}
Warehouse or distribution centre managers have to decide how to collect the products to fulfill customers requests but also where to locate the products (SKUs) and how much space to allocate to each of them. Moreover, they have to deploy replenishment strategies to guarantee the reliability of their own stocks. These are challenging decisions because of their level of complexity and their high impact on the centre performance in terms of both its throughput and the operation costs. In particular, the goal of this work is to evaluate whether specific strategies to share the storage space could lead to reduce the operation costs while keeping the service level as high as possible. To this end, this paper develops a discrete event simulation model of the logistic operations at a real high throughput warehouse which handles more than 12 millions of cases annually. Preliminary results show that potential economies may be achieved by reducing the number of stock-outs at the picking area where customer orders are collected.
\end{abstract}

\section{INTRODUCTION}

Although a large number of research works concerns supply chain simulation, considerable less effort has been concentrate in warehouse or distribution centre operations, a key element in supply chain efficiency. Operations at such facilities may be roughly separated into storing and picking. Inbound products coming from external or internal manufacturers are received, sorted and stored. On the order hand, according to client requests products are collected at the store by employees (the pickers) and moved to the transportation docks prior to distribution. Storage decisions concern mainly the assignment of SKUs among the available storage locations. Classic storage strategies include dedicated storage, in which products are allocated to fixed locations; random storage, in which products are allocated to various locations according to the available storage space; and classed-based storage, in which products are al- located to specific zones or areas in the warehouse. These basic strategies may be combined to better respond to specific requirements. Since the number of locations is often limited, such decisions implicitly require determining the number of locations assigned to each product, whose impact on the warehouse performance will be deeply discussed in this paper.

Order picking strategies determine how the ordered SKUs are grouped into picking lists, and subsequently retrieved from their storage locations by one or many pickers. There are four basic procedures for picking orders: discrete, zone, batch and wave. In discrete picking, one person picks one order, one line at a time. This strategy is often preferred because it is easily implemented and order integrity is always maintained. In zone picking, the warehouse is divided into distinct zones, with one picker assigned to each zone. This means that the items in an order are divided into several picking lists. In batch picking, one person may pick many orders at the same time. In wave picking, orders are picked to satisfy the required shipping schedule. All other picking practices are a combination of these basic procedures.

Operating DCs or warehouses requires much more than choosing storage and picking strategies. In fact, even given a basic storage model-dedicated storage, for example - many decisions must be made: what type of equipment should be chosen (e.g., racking), whether certain equipment (e.g., handling devices like conveyors) should be used, and which products should assigned to which storage locations. The goal of these decisions is to allow warehousing systems to respond rapidly to customer demands, and the choices made play an important role in the success of a supply chain. However, these challenging decisions are very difficult to make and, as will be shown in the literature review section, available analytical models such us inventory models - cover only specific parts of the problem. Therefore the aim of this paper is to provide an operation oriented simulation model that could help managers assessing the performance of the different storing and 
picking strategies. In particular, we think that optimizing the assignment of storage locations (the number of slots) to products could also contribute to improve operations. As a first step towards the design and implementation of a decision support system, a simulation model integrating different assignment and replenishment strategies and allowing managers to choose among them was developed.

This paper is structured as follows. The next section presents a brief literature review. Section 3 describes in detail the industrial setting here considered and the logistic operations deployed at the warehouse. Section 4 focuses in the simulator development and specifies the implementing choices made. Section 5 is devoted to the simulation execution and results analysis. Conclusions and further research avenues are reported in Section 6 which concludes the paper.

\section{BRIEF LITTERATURE REVIEW}

Most of the research concerning warehouse operations has concentrated either in order picker routing (determining the minimum distance picker route within a warehouse is a variant of the classic Traveling Salesman Problem, which is NP-hard, Laporte 1992) or product location strategies. Product location strategies (i.e. where to store products in order to facilitate order picking) has received considerably less attention. In his classic work, Heskett (1963) developed the cube-per-order index, in which the most popular products are located closest to the base location. This index minimizes the routing distance for cases in which one product is picked on each route (pallet picking). Jarvis and McDowell (1991) developed a model for locating products in a warehouse in such a way that the average orderpicking time is minimized. Recently, Jewkes, Lee and Vickson (2004) took a closer look at product location along a picking line. In their study, each order was filled by moving a container past the various product storage locations, with the appropriate quantity of each product being transferred from its respective storage location to the container. To the best of our knowledge, the problem of product location involving a conveyor belt, like the one studied in this paper, has never been addressed before.

On the other hand, simulation has traditionally been one of the most popular approaches to investigate warehouse operations. From the pioneers research works underlining the potential of simulation in warehouse management (see for example Bafna, 1973 and Biles 1977) to the most recent publications, simulation has mostly been used as a means to validate the performance of warehouse designs or material handling devices. In particular, most of the OR models and results reported in the previous subsections were validated upon simulation approaches. Examples of such a mixed optimization-simulation approach are found in Gue et al. (2006) and Renaud and Ruiz (2007). However, despite of the large number of papers in the lit- erature, theoretical or general contributions are scarce, given that product location is highly dependent on warehouse configuration and on the order-picking strategy and technology in place, and thus most of the research in the literature deals with specific practical cases.

\section{THE WAREHOUSE OPERATIONS}

The company studied in this paper is one of the largest snack selling company in the United States (potato chips, corn chips, hardy pretzels, savory cookies, ...). The facility under study is its second largest production plant in Canada, producing annually more than 40 millions of kilograms of products. More than 12 millions of cases are handled annually by the distribution center, which shows two different storage areas depending on the type of customer to be served (see Figure 1). The first type of customer includes local DCs and/or internal customers. These customers order large quantities of products which are shipped on pallets by truckloads. Orders are assembled using a pallet picking strategy, with forklifts moving back and forth between the warehouse, where the pallets are stored on standard racks, and the shipping area, where the trucks wait to be loaded. This section of the warehouse has adopted a random storage policy.

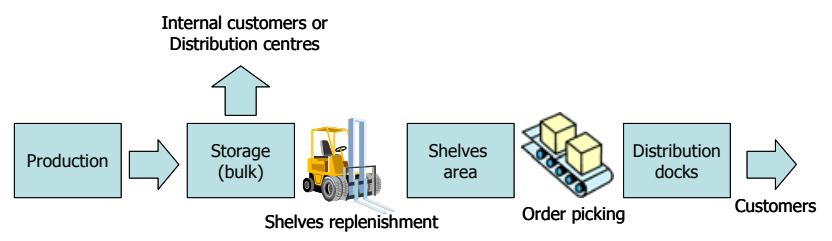

Figure 1: Warehouse operations

The second type of customer comprises external retailers, who may order any quantity of any product. These customers generate the greatest part of the orders. Products for these customers are assigned to storage locations (slots) in a shelving system along a conveyor belt, according to a dedicated storage policy (one kind of product by slot). A wave discrete order picking policy is used. The orders that have to be loaded in a given truck are released (waving) following the shipping schedule. Thus, the picker works on a single order (discrete picking) walking along the shelves, gathering the required quantities of the products from the various locations along the route (case or box picking), and putting them on the conveyor belt. Once the picker has completed the order, she triggers an acknowledge signal at the end of the conveyor which starts moving the items products to the right truck in the shipping area at the time that the picker returns to the beginning of the shelving system. As orders are picked one by one, there is no need to sort the products. The average throughput of this picking system is around 600 cases per hour and it works 24 hours a day. A more detailed description of the warehouse layout 
and, in particular, of the shelves and picking areas can by found in Gagliardi, 2006.

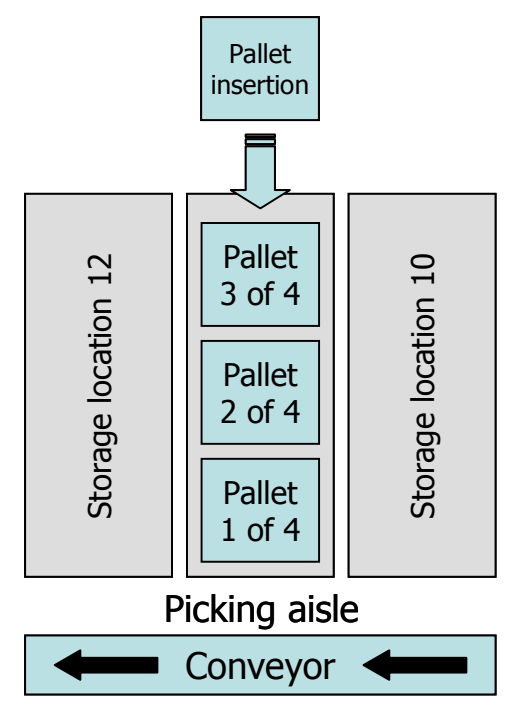

Figure 2 : The shelves and picking area

Shelves are replenished from the bulk part of the warehouse by means of a forklift operated by a single employee. She drives along the back side of the shelves and whenever the stock level of a product seems low enough, she decides to refill the shelf. The refilling cycle consists in driving to the bulk area, locate the product and pick a pallet. The pallet is then moved to the shelving area and placed at the targeted slot.

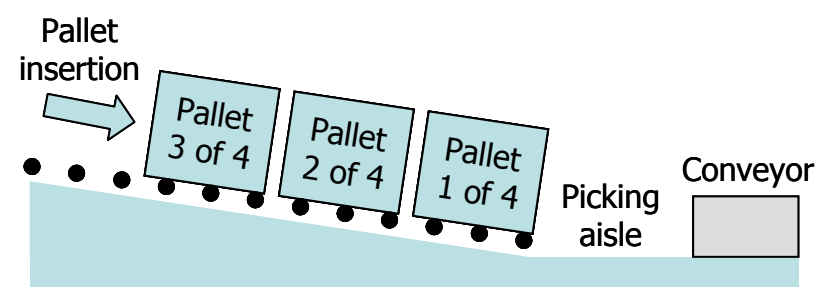

Figure 3 : The shelves replenishment

This paper focuses exclusively on the shelving system used to service the latter category of customer. In particular, the company has observed in the last months that the performance of picking operations has decreased. In fact, it often happens that a picker has to wait in front an empty shelf till the product is replenished, increasing the time of preparation of an order and thus reducing the number or orders produced per day. Therefore, it happens that the company has to call for extra time in order to fulfill the customers requests and that distribution trucks must wait at the loading decks. In order to balance the product flow at the shelving system, all the locations are refilled during the weekend, so every Monday morning inventories are full and the system is ready to begin the new week.

\section{MODELING THE PICKING ACTIVITIES}

Let us assume that a list of orders to collect and a set of parameters describing the system configuration are provided. Among the former, let us mention the assignment of storage locations in the shelves area to products, the picker walking and handling speed, and the replenishment cycle length. Let also assume that the orders are listed preprocessed in the following way. First, the orders from customers being visited by in the same truck are grouped and second, orders are sorted in the inverse order in which customers will be visited. Moreover, each order contains a given number of lines. A line specifies a required product and the quantity to pick. Lines in an order are also automatically sorted according to the shelves assignment, the first product in the list being the closest one to the picking starting point.

Orders are sequentially picked. Picking is modelled by two activities : walking and handling (grab the boxes from the shelf and put them on the conveyor). Walking time is assumed to be constant for every order as the picker must arrive till the end of the shelves area before come back to the starting point. Handling time depends on the number of items to "launch" on the conveyor. Therefore, whenever a new order is selected to be collected, the simulation algorithm verifies the inventory status of each product for each line and, if the whole order can be fulfilled, then the end of the picking is automatically estimated by adding the walking time to the handling time computed by multiplying the number of items by the launching time. Then, the inventory for each product is updated and the algorithm selects the following order to be picked.

Unfortunately, it happens that the quantity required by a line can't be satisfied by the inventory on the shelves. In this case, we estimate the picker arrival time to the location of the stock-out product. This is done by adding the launching time of the previous lines in the order and the walking time to the location of the stock-out product which is estimated by dividing the total walking time by the number of lines to be picked (this produces a sort of "average time" between the storage locations to be visited) and multiplying it by the order of the stock-out product in the picking list. If a replenishment of the product has been done from the moment that the picker start the collection, the picker launches the line and the time to complete the rest of the order - or to move towards the next stock-out product - is computed. However, if the location has not been replenished, the picker lunches partially the line with the remaining boxes on the shelf after what she must wait till the shelf replenishment prior to complete the line and move to the next location.

The picking activities as described in the previous paragraphs were validated with the warehouse operations manager in order to ensure that our perception of the tasks and the relationships among them fitted the warehouse re- 


\section{Gagliardi, Renaud and Ruiz}

ality. The implementation of the simulation model is thoroughly described in the next section.

\section{IMPLEMENTING THE MODEL}

Any computer based simulator aims to truthfully mirror the behaviour of the real system. To achieve this goal, the different decision processes along the operational cycle described in the previous section must be accurately reproduced. Keeping this in mind, the simulator presented here is built around a discrete event simulator engine (DES) using the three phases approach (Pidd, 1995). Discrete event simulation concerns the modelling of a system as it evolves over time by a representation in which system variable changes instantaneously at separate points in time - the ones in which an event occurs (Law and Kelton 2000). Basically, the simulator contains three main components : a database, a list of events, and a simulation clock that manages the simulated time.

The data base contains the list of orders to be processed and it also stores the system parameters. Presently, the data base is feed exclusively with historical data provided by our industrial partner, but pseudo random data is expected to be generated soon after conducting statistic input modelling tests on the real data. Variates obtained from such a pseudo random generator will allow assessing robustness and test specific situations or scenarios.

The simulation engine contains basically a list of events and a simulation clock that advances the simulated time. The events list is a chronologically ordered list containing the time when each type of event will occur. Events list is sequentially examined and, each time an event is processed, future - either conditional or bounded - events are created and added to the event list. After executed an event, it is deleted from the list and the clock is advanced to the following event. The warehousing activities here studied are modeled by means of three different events. At the beginning of the simulation, the events list contains only two events : an event triggering the picking of the first order in the order's list, and an event associated to the beginning of the first replenishment activity. Therefore, the simulation clock - set to zero at the beginning of the simulation - is advanced to the first of these events and the event is executed. For example, let us assume that the first event in the list is $\mathrm{SPO}_{1}$ (start picking order 1) at time $\mathrm{t}_{1}$. Executing $\mathrm{SPO}_{1}$ consists in checking the stock for all the lines in the first order and, if every line can be fulfilled, create a $\mathrm{SPO}_{2}$ (start picking order 1$)$ event at time $\mathrm{t}=\mathrm{t}_{1}+$ walking time + handling time, computed as described in the previous section. Then, $\mathrm{SPO}_{1}$ is deleted from the list and the clock is advanced to the following not executed event (either $\mathrm{RP}_{1}$ or $\mathrm{SOP}_{2}$ ). If a stock-out is detected during the inventory check, a PP (partial picking) event is created at the time the picker is expected to arrive at the stock-out location (see the previous section) instead of a SPO. When a PP event is executed, the inventory at the location is checked. If the inventory fulfills the request, either a SPO if the rest of the lines in the order can be collected - or another PP at the next stock-out location is created and added to the event list. However, if the inventory does not fill the line, another PP event at the same location is created just after the following replenishment event. This modeling approach, with respect to the one generating a new event each time the picker moves from one storage location to the next, speeds up the simulation execution reducing the number of read/writing access to the events list when the order can be collected without stock-outs (most of the cases). On the other hand, the picker arrival time to a stock-out location is roughly approximated.

The execution of a replenishment event creates a new replenishment event at time $t=$ present time $+t_{r}, t_{r}$ being computed from a normal distribution whose parameters were fit to the historical replenishment times observed in practice. When generating a new replenishment event, one has also to decide which product to refill. Several strategies or selection rules are available according to information considered to make the decision and the desired level of synchronization between picking and replenishment. For example, a pure random rule would chose with equal probability among the storage locations that can be refilled i.e. there is at least free space as to add a full pallet of product. Clearly such a rule presents no synchronization at all between picking and replenishment activities and it was discarded after consulting replenishment employees. The most intuitive yet simple rule consisting in replenish first the product having the lowest inventory level was finally implemented.

Needless to say, the data base stores all the event executions, allowing further results and performance analyze or even to review a whole simulation of the different decisions made along the simulated period. Finally, parameters concerning the simulation experiment itself such as the number of replications or the length of the simulation are also stored in the database.

The simulation implementation has been coded in VB.net (Visual Basic 7.0). The code was debugged by means of the associated Microsoft tools, in particular the step-by step running mode that allowed us to validate the code by following the algorithmic flow.

The choice of VB.net was based on two arguments. First, programming languages are more flexible than standard simulation software. Since our research aims at testing a large number of location and replenishment strategies, the model implementation ought to accept such modifications dynamically. Doing so with simulation software as ARENA or VISUAL8 would certainly required us to use the embedded programming code - i.e. SIMAN in the case of ARENA - which represents as much work as coding directly in programming languages as VB.net. Secondly, VB.net was chosen among other languages $(\mathrm{C}++$ or 
Java) because it was the language already used by our partner developers. In addition, we found that VB.net is supported by a worldwide community of developers who contribute useful banks of libraries that made the implementing of simulation models easier than expected.

\section{NUMERICAL EXPERIMENTS AND PRELIMINARY RESULTS}

This section aims at proving that the allocation policy which shares the available storage locations among the products has an impact on the warehouse performance. To this end, real data provided by our industrial partner has been feed into the simulator in order to compare the performance of four different allocation rules.

\subsection{Experiment setting}

The simulator will reproduce the picking of 58149 lines corresponding to the company's demand during eight consecutive weeks. The demand concerns 253 different products which has to be assigned to the 1012 available storage locations. Recall that (1) commands are picked one at a time and (2) the picker must walk the entire storage area at each command picking. Then, to address the goal of this study, we only need to determine the number of locations reserved for each product (how many) rather that their specific physical location (where). Moreover, since at this point our main interest centers exclusively in evaluating the impact of different space allocation strategies with respect to the system efficiency, walking and launching times have been assumed to be constants despite of their (low) variability.

Space allocation is done by a simple priority based assignment algorithm which proceeds as follows. At the beginning, the algorithm assigns one single slot (location) to each product in order to ensure that all the products will be present on the storage zone. Then, it assigns one slot to the product having the highest dispatching ratio, computes the product's new ratio and keeps assigning slots till all of them have been assigned. In particular, four dispatching rules were used to generate four different allocation scenarios. The first scenario gives equal priority to all the products, leading to an homogeneous storage space assignment. On the other hand, the other scenarios aim at assign more locations to the most popular products, the popularity of each product being estimated differently by each rule, while the remaining rule

Rule 1 : Homogeneous sharing. This scenario shares the allocations among the products in an homogeneous manner. Thus, since 1012 locations must be shared among 253 products, each product occupies four locations.

Rule 2 : Product demand frequency. This scenario shares the allocations among the products according to their popularity, which is estimated by dividing the number of commands containing the product by the total number of commands considered and by the number of storage locations that it has received so far.

Rule 3 : Product demand quantity. This scenario shares the allocations among the products according to their popularity, which is estimated by the total product demand over the eight weeks considered divided by the number of storage locations that it has received so far.

Rule 4 : Product picking quantity. This scenario command shares the allocations among the products according to the size of its average pick, which is estimated by dividing the total product's demand by the number of commands requiring the product and by the number of storage locations that it has received so far.

Our preliminary tests are based on real data provided by the company exclusively. Statistical analyze of demand is being currently conducted in order to model demand patterns and behavior but the results of this study are not available yet. Nevertheless, we run eight experiments, each using the company's demand for one week. Although robustness and variance analyze can not be performed on such a small experiment setting, al least it will allow us assessing the potential of our approach.

The system here studied is clearly a terminating one, since the shelving area is fully replenished at the end of each week. Furthermore, the same initial condition in which every product inventory is filled up to its assigned capacity applies. Finally, we set the walking speed and the replenishment time, $t_{r}$, to constant values suggested by our industrial partner.

\subsection{Preliminary results and discussion}

As we mentioned in the previous sections, the primary goal of the company is to increase the efficiency of the order picking process by reducing the picker's idle time caused by stock-outs at the shelves. Thus, Table 1 reports the results of our experiments. For every assignment strategy, the number of stock-outs observed at each experiment are reported. Also, lines Total and Gap provide the total number of stock-outs over eight consecutives working weeks and the difference (in percentage) with respect to the best rule, respectively.

First of all, we were extremely surprised by the number of stock-outs and therefore by the amount of idle time reported. Let us recall that the average replenishment cycle requires two minutes which means, assuming 200 stockouts, more than 6.67 hours of idle time per week.

Since the average results in Table 1 doe not show a clear dominance between the space allocation strategies under test, we also computed the $95 \%$ confidence intervals for the results obtained for each rule. Unfortunately, the widths of such intervals are too large as to statistically conclude on the performance of the four rules, as reported by Table 2. 
Table 1 : Experiments results

\begin{tabular}{|c|c|c|c|c|}
\hline Week & Rule 1 & Rule 2 & Rule 3 & Rule 4 \\
\hline $\mathbf{1}$ & 108 & 147 & 106 & 99 \\
\hline $\mathbf{2}$ & 159 & 180 & 164 & 177 \\
\hline $\mathbf{3}$ & 177 & 197 & 181 & 177 \\
\hline $\mathbf{4}$ & 154 & 181 & 152 & 161 \\
\hline $\mathbf{5}$ & 187 & 208 & 161 & 179 \\
\hline $\mathbf{6}$ & 214 & 240 & 224 & 214 \\
\hline $\mathbf{7}$ & 205 & 226 & 182 & 192 \\
\hline $\mathbf{8}$ & 196 & 241 & 204 & 220 \\
\hline \hline Total & $\mathbf{1 4 0 0}$ & $\mathbf{1 6 2 0}$ & $\mathbf{1 3 7 4}$ & $\mathbf{1 4 1 9}$ \\
\hline Gap & $\mathbf{1 , 8 9 \%}$ & $\mathbf{1 7 , 9 0 \%}$ & $\mathbf{0 , 0 0 \%}$ & $\mathbf{3 , 1 7 \%}$ \\
\hline
\end{tabular}

Table 2 : One sample test (T-test)

\begin{tabular}{|c|c|c|c|}
\hline & & \multicolumn{2}{|c|}{ 95\% Confidence Interval } \\
\hline & Average & Lower & Upper \\
\hline Rule1 & 175 & 146,3932 & 203,6068 \\
\hline Rule2 & 202,5 & 175,0437 & 229,9563 \\
\hline Rule3 & 171,75 & 142,0246 & 201,4754 \\
\hline Rule4 & 177,375 & 146,1165 & 208,6335 \\
\hline
\end{tabular}

Despite of the large variability of these results, we performed paired samples tests for all the combinations of rules taken two by two. The tests showed that rule 2 is dominated by rules 1,3 and 4 but no other result could be stated. Clearly, more replications are required in order to obtain more accurate results but we can conclude that the location sharing strategy impacts the system performance (or at least that bad strategies can deteriorate it!). Furthermore, although picking and replenishment operations seems to be independent, we think that a decision support system synchronizing order release and replenishments could help increasing the performance of the warehouse reducing the number of stock-outs on the shelves. Since the list of orders to prepare is known a priori, we think that replenishments could be planned in a proactive rather than reactive mode. Forthcoming research will be oriented towards the design of replenishment algorithms able to look forward into the entire list of commands in order to minimize the number of stock-outs.

\section{CONCLUSIONS}

This paper presents a discrete event simulation model of the internal logistic operations at a real high throughput warehouse. The model focuses in the order picking and shelves replenishment processes and, as a first application, it is used to discuss the impact of storage space allocation on the system performance. The modeling approach adopted can easily accept slight modifications to account for particularities of other conveyor based industrial settings. Four storage space sharing rules are proposed and compared. Eight working weeks were simulated. The results show large variability which does not allow to identify a dominant rule. However, despite such large confidence intervals, we can observe that rule 2 is outperformed by the other rules. To sum up, we showed that space sharing rules impact the system performance but additional experiments based on demand replication are required to reduce the results variability. Also, more research is needed to better understand the system dynamics and, in particular, the links between order picking and replenishment decisions. We think that the simulation platform will allow us conducting such future research.

\section{REFERENCES}

Bafna, K.M. 1973. A Simulator for Designing High-Rise Warehouse Systems, Proceedings of the 1973 Winter Simulation Conference San Francisco, 128-138.

Biles, W.E.1977. Computer simulation and material handling systems. Material Handling Industry of America, Charlotte, NC.

Gagliardi, J. P. 2006. Simulation et optimization de cueillettes sur convoyeur. M.Sc. thesis, Département Opérations et systèmes de decision, Faculté des sciences de l'administration, Université Laval.

Gue K.R., R.D. Meller and J.D. Skufca. 2006. The effects of pick density on order picking areas with narrow aisles. IIE Transactions, 38, 859-868.

Heskett J.L. 1963. Cube-per-Order index - A key to workhouse store location. Transportation and Distribution Management, 3, 27-31.

Jewkes E., C. Lee and R. Vickson. 2004. Product location, allocation and server home base location for an order picking line with multiple servers. Computers and Operations Research, 31, 623-636.

Laporte, G. 1992. The traveling salesman problem: An overview of exact and approximate algorithms. European Journal of Operational Research, 59, 231-247.

Law, A. M., and W. D. Kelton. 2000. Simulation modeling $\&$ analysis. 3rd ed. New York: McGraw-Hill, Inc.

Pidd, M. 1995. Object orientation, discrete simulation and the three-phase approach. Journal of the Operational Research Society, 46, 362-74.

Renaud, J. and Ruiz, A. 2007. Improving Product Location and Order Picking Activities In a Distribution Center, Journal of the Operational Research Society, forthcoming. 


\section{AUTHOR BIOGRAPHIES}

JEAN PHILIPPE GAGLIARDI is a PhD student in the department of Operations and Decision Systems at Laval University. His research interest lies in the area of Manufacturing and Operations Management and more specifically, in modern warehouse and distribution systems simulation and optimization. $\mathrm{He}$ is a student member of the Interuniversity Research Center on Enterprise Networks, Logistics and Transportation (CIRRELT).

JACQUES RENAUD is professor in Operations and Decision Systems at Laval University. Member of the Interuniversity Research Center on Enterprise Networks, Logistics and Transportation (CIRRELT), his research interest lies in the area of transportation, vehicle routing, warehousing and production planning. His web page is at <www. fsa.ulaval.ca/personnel/renaudj/>.

ANGEL RUIZ is professor in the department of Operations and Decision Systems at Laval University. He holds a $\mathrm{Ph} . \mathrm{D}$. from the Université de Technologie de Compiègne (France). His current research interests are in supply chain management, logistics and computer simulation applied to solve production planning and control optimization problems. He is member of the Interuniversity Research Center on Enterprise Networks, Logistics and Transportation (CIRRELT). 